\title{
Infective Endocarditis: The Anesthesiologist's Perspective
}

\author{
Usha Kiran, Arindam Choudhury, Neeti Makhija, Kulbhushan Saini, Randhir Singh Rajput, Balram Airan
}

\begin{abstract}
Infective endocarditis (IE) usually involves one or more heart valves resulting in vegetation, perforation, abscess, fistula and/or pseudoaneurysm formation. In IE, the mainstay of treatment remains antimicrobial agent for 4 to 6 weeks. However, 40 to $60 \%$ requires surgical intervention because of some complications. Surgery could be risky in the active stage of the disease, but it becomes essential to avoid preoperative heart failure, irreversible structural damage and also to prevent systemic embolization. Perioperative transesophageal echocardiography (TEE) examination is an important diagnostic modality for guiding therapeutic decisions. We recently managed four patients of IE. Two of them presented with mitral valve vegetations resulting in severe mitral regurgitation and one with aortic root abscess. We monitored TEE in all the four cases throughout the surgery.
\end{abstract}

Keywords: Infective endocarditis, Vegetation, Aortic root abscess, Transesophageal echocardiography.

How to cite this article: Kiran U, Choudhury A, Makhija N, Saini K, Rajput RS, Airan B. Infective Endocarditis: The Anesthesiologist's Perspective. J Perioper Echocardiogr 2013; 1(2):57-61.

\section{Source of support: Nil}

Conflict of interest: None declared

\section{INTRODUCTION}

Infective endocarditis (IE) normally presents as valvulopathy producing regurgitation or stenosis of one or more heart valves. Vegetations, abscess, fistulae or pseudoaneurysms in and around the heart valves or other prosthetic materials are not uncommon in IE. Transesophageal echocardiography (TEE) is very useful inside the operation room as it delineates abnormal from the normal anatomy of the heart and is a very essential monitoring tool for patients with IE.

\section{CASE REPORTS}

\section{Case 1}

A 45-year female was hospitalized with diagnosis of rheumatic heart disease (RHD), severe aortic regurgitation (AR), infective endocarditis (IE) with aortic root abscess, severe LV dysfunction and type 2 diabetes mellitus (DM). She had history of chills and rigor 6 months back for which she had received broad spectrum antibiotic for 1 month. Blood cultures were sterile at the time of admission. Patient was receiving digoxin, spironolactone and metformin.

She was induced with fentanyl, etomidate and rocuronium under monitoring as per ASA standards. After induction of general anesthesia, intraoperative transesophageal echocardiography (TEE) in mid-esophageal (ME) aortic valve (AoV) short axis and aortic valve long axis view revealed a $3 \times 8 \mathrm{~mm}$ abscess cavity adjoining the right coronary cusp below the annular plane of AoV extending into the muscular interventricular septum (Figs 1A to C). The native infected valve was replaced with a $19 \mathrm{~mm}$ tissue (Carpentier-Edward bioprosthetic) valve under cardiopulmonary bypass (CPB). Post-AoV replacement, TEE revealed the prosthetic valve was functioning well, the abscess cavity was obliterated and there was no paravalvular leakage. Postoperative recovery was uneventful, she continued to receive antibiotics and subsequently, she was discharged on 8 th postoperative day.

\section{Case 2}

A 35-year female with a diagnosis of RHD, severe mitral regurgitation (MR), severe tricuspid regurgitation (TR) with

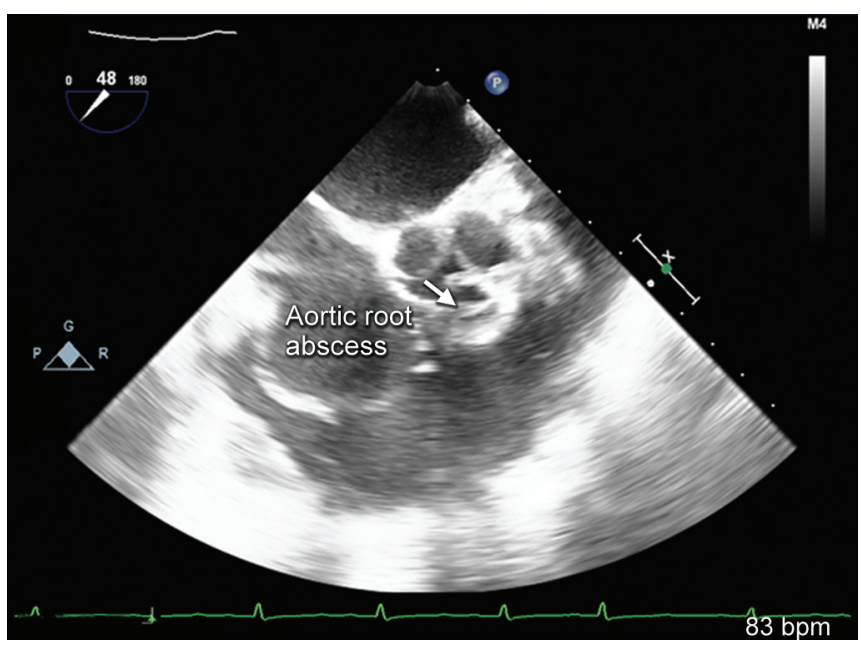

Fig. 1A: Mid-esophageal (ME) aortic valve short axis (SAX) view showing aortic root abscess

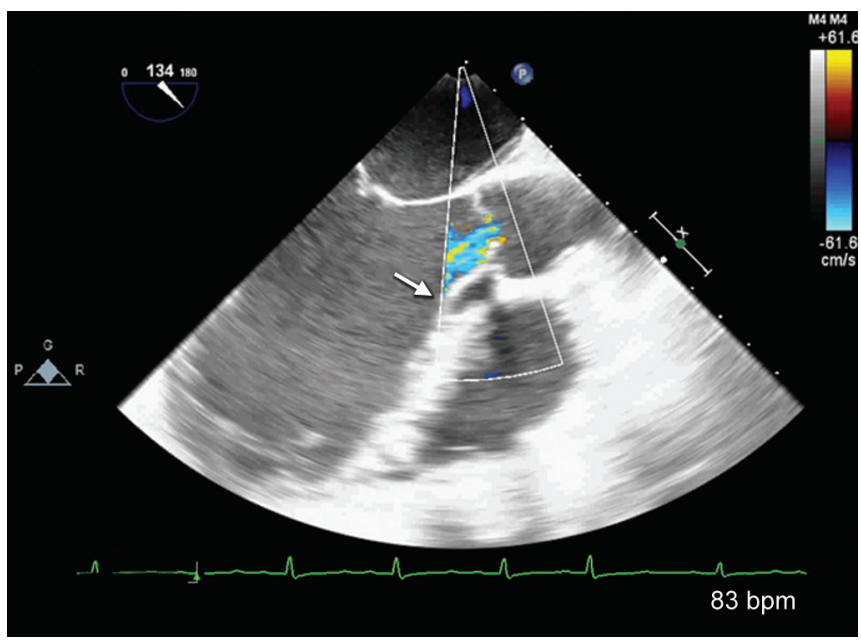

Fig. 1B: ME aortic valve long-axis (LAX) view showing aortic root abscess 


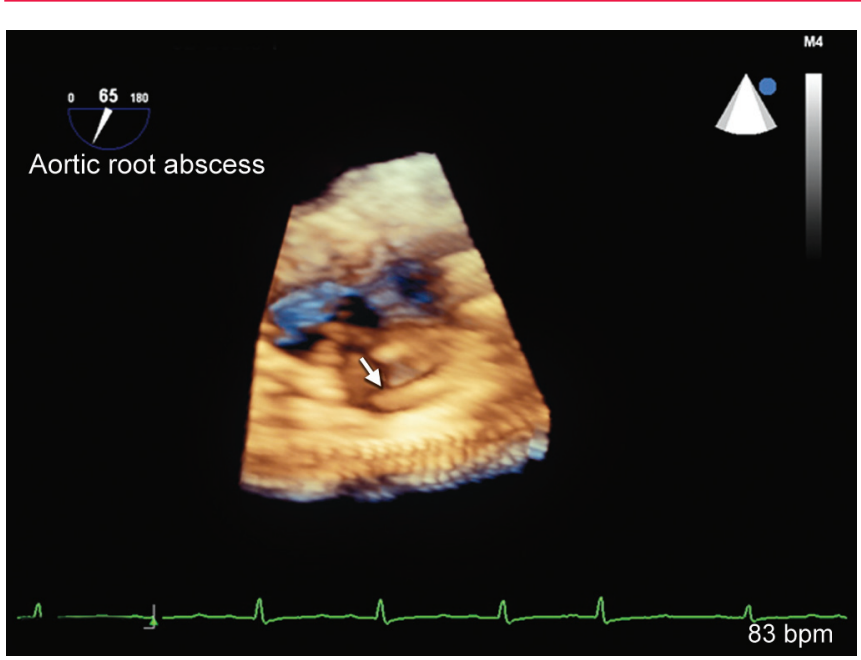

Fig. 1C: 3D still frame showing abscess cavity adjacent to RC cusp of the aortic valve

IE of mitral valve with left ventricular ejection fraction (LVEF) of 30\% presented for valvular heart surgery. Six weeks ago, she had history of high grade fever with chills and rigor. Her hemoglobin $(\mathrm{Hb})$ was $9 \mathrm{gm} / \mathrm{dl}$, total leukocyte count (TLC) was $13000 / \mathrm{cmm}$, blood urea was $80 \mathrm{mg} \%$ and creatinine was $1.2 \mathrm{mg} \%$. Blood cultures were sterile. Her abdominal ultrasonogram (USG) revealed congestive hepatomegaly. She received intravenous antibiotics for 4 weeks without improvement in her clinical condition. However, the patient improved symptomatically with tablet digoxin and spironolactone. She was posted for mitral valve replacement and tricuspid valve repair. After induction of general anesthesia, intraoperative TEE revealed severe MR (Carpentier type II) due to prolapse of anterior mitral leaflet. Two mobile vegetations were also seen on the free edge of mitral valve leaflet (Fig. 2). Examination of tricuspid valve showed severe TR due to tricuspid annular dilation, annulus was $6.4 \mathrm{~cm}$ in diameter when measured in four-chamber (4C) view. There was no tricuspid stenosis. Mitral valve

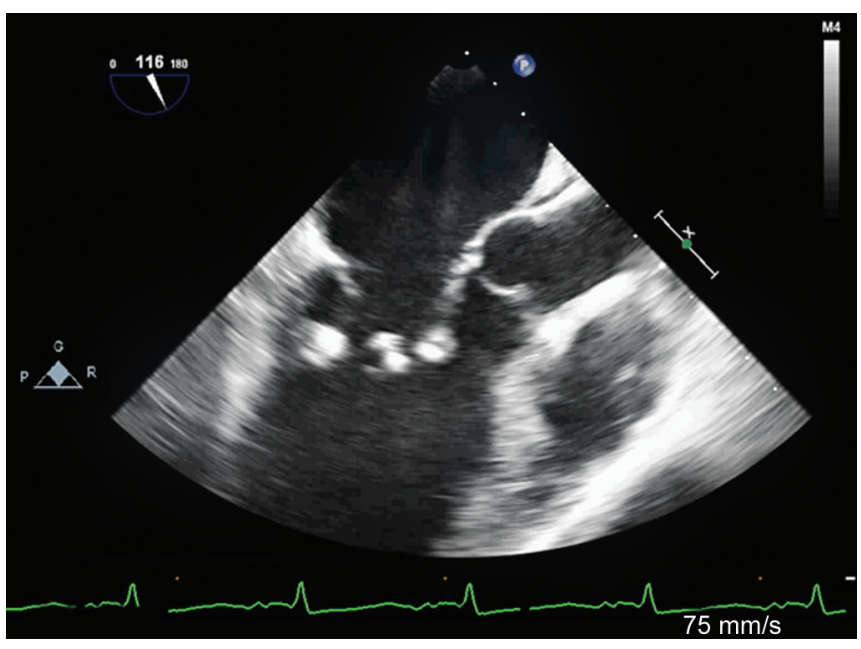

Fig. 2: ME AoV LAX view shows vegetations on both the cusps (anterior and posterior mitral leaflet) replacement (MVR) and tricuspid valve repair were done under cardiopulmonary bypass (CPB). Post-CPB, TEE examination revealed no paravalvular leak, gradient across the prosthetic valve was within the normal range and leaflet movements were absolutely normal. Perioperative course was uneventful.

\section{Case 3}

A 30-year female with RHD, severe MR, mild TR, severe pulmonary artery hypertension (PAH), IE of the mitral valve with type 2 diabetes mellitus (DM) presented with dyspnea on exertion and fever for 1 month, pain in the lower limb for 15 days as she had an embolic episode during that time. She received broad spectrum antibiotics for 1 month and became afebrile after 7 days of treatment. Her blood culture at admission was sterile. The BUN was 60 to $90 \mathrm{mg} \%$ and creatinine was 2.2 to $2.9 \mathrm{mg} \%$. USG abdomen was suggestive of renal parenchymal disease. After induction of general anesthesia, intraoperative TEE exam confirmed RHD with severe MR (Carpentier type II), mod TR with predicted right ventricular systolic pressure (PRVSP) of $84 \mathrm{~mm} \mathrm{Hg}$. The PRVSP was derived from the following formula; $\mathrm{PRVSP}=\mathrm{TR} \Delta+\mathrm{RAP}=72+12=84 \mathrm{~mm} \mathrm{Hg}$ suggestive of severe PAH. There were hypermobile vegetations on mitral valve (AML), the largest one measuring $22 \times 12 \mathrm{~mm}$ (Fig. 3). She underwent MVR under CPB. Her intraoperative course was uneventful. On the 2 nd postoperative day, she was re-explored for pleura-pericardial temponade and 2 liters of clot were removed from the left pleural cavity. No obvious surgical bleed was detected.

\section{Case 4}

A 40-year-old male patient with the diagnosis of pseudoaneurysm of the aortic root and severe AR was

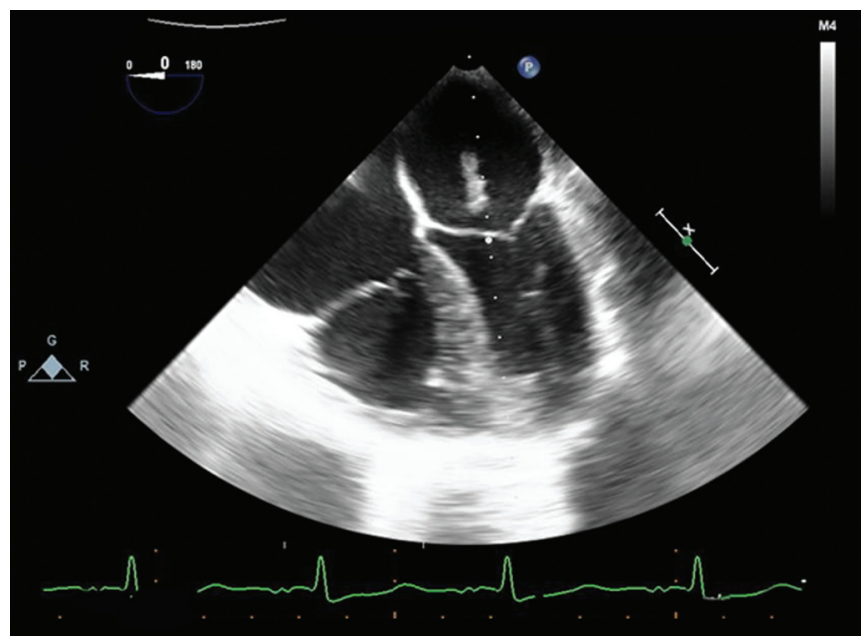

Fig 3: ME 4C view showing large hypermobile vegetation on the AML 
scheduled for surgery. There was a history of previous two hospitalizations; first was 9 months back with fever and palpitation, and second was 3 months before current admission for palpitations and dyspnea on exertion. After induction of anesthesia, TEE examination in ME AoV long axis view showed severe AR on color flow Doppler. There were two regurgitation jets, one from a perforated aortic cusp and other from the aneurysmal sac into the left ventricular outflow tract. The diameter of the aortic annulus measured $3.08 \mathrm{~cm}$. There were no vegetations seen on aortic leaflets. An aneurysmal sac was seen as an echo-free space from basal interventricular septum extending into aortic root ME AoV LAX view (Fig. 4). ME AoV SAX view also revealed a diastolic in coaptation defect of the $\mathrm{AoV}$ and an echo-free space of the aneurysmal sac in the annulus extending into the right coronary sinus.

\section{DISCUSSION}

All the four cases presented with heart failure after receiving broad spectrum antibiotic cover with minimally compromised renal function. These cases carry a higher risk of perioperative morbidity. Careful intraoperative management with hemodynamic stability was the primary anesthetic goal. Therefore, etomidate with fentanyl were used for induction of anesthesia. All aseptic precautions were implemented as this subset of patients pose high risk for prosthetic valve endocarditis. Antibiotic were continued postoperatively in the cardiac surgical ICU. Till date, there are no clear guidelines for anesthesiologists for the management of patients with IE undergoing vegetectomy and/or heart valve replacement.

The role of perioperative TEE in the medical and surgical management of this subset of patients is of paramount importance. Intraoperative TEE determines the exact location of vegetations/abscess and guides the surgeon

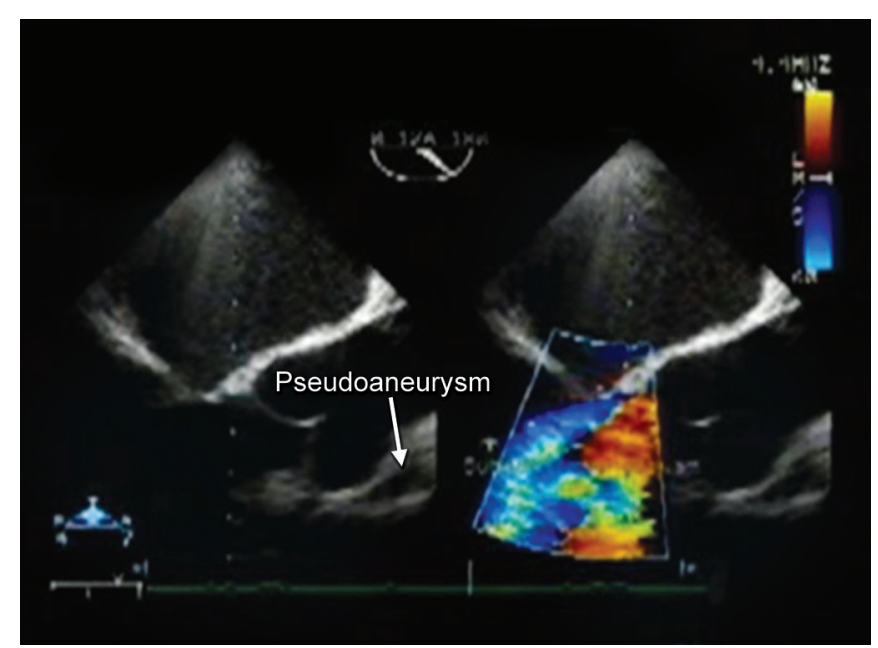

Fig. 4: ME aortic valve LAX view showing paravalvular pseudoaneurysm formation whether the valve is to be replaced or repaired and also while coming off bypass to have a postoperative assessment of the surgical techniques offered to the patient. ${ }^{1}$ In all patients described in our case series, valves were replaced. In two of our patients bioprosthetic valves were used. The literature suggests the use of homografts or tissue valve to reduce the risk of recurrent infections. ${ }^{2}$

The echocardiographic diagnosis of IE requires a comprehensive multiplane (i.e. different views in TEE) evaluation of all valves and associated tissues. Evaluation also includes the tissues surrounding any valve with suspicious findings, like leaflet perforation, fistula, abscess, echo-free space, etc. During intraoperative TEE examination, one should not forget to evaluate the Chordae tendiniae and other supporting structures of the atrioventricular valves. Comprehensive Doppler echocardiographic examination is also an essential component of this evaluation. Vegetations are the hallmark of diagnosis of IE. They appear as echodense intracardiac masses on the valve or their supporting structures. The examination should describe the size, shape, mobility, number and location of the vegetations. Larger size and greater mobility of vegetations increases the likelihood of systemic embolization. Vegetations are common in the low pressure side of the regurgitant valve, i.e. ventricular side of the aortic valve or left atrial aspect of the mitral valve. The other characteristic feature of vegetations that might help in echocardiographic detection is that there motion is chaotic and independent of cardiac cycle. At times, they may be found in the chamber wall and their sizes vary from 0.1 to $1 \mathrm{~cm}$.

The Duke's diagnostic criteria also recommended TEE based positive detection (of oscillating mass on valve or supporting structures, on the implanted material, or periannular abscess or new partial dehiscence of prosthetic valves) as major criteria apart from clinical and microbiological criteria. ${ }^{3}$ Perivalvular cavities (echofree spaces) form when annular infection breaks through the weakest point and spreads into the contiguous tissues surrounding it. In native AoV endocarditis, this generally occurs through annulus near the membranous septum and the AV node. The anatomic vulnerability explains why both abscesses and heart blocks are frequent sequelae in this condition. ${ }^{4}$ Due to systemic intravascular pressures, abscesses may progress to fistulous tract that may create intracardiac or pericardial shunts. Such lesions are not amenable to medical management alone and needs urgent operative intervention. TEE is the modality of choice for assessment of any patient at risk of perivalvular extension of IE. ${ }^{5,6}$

Guidelines on antibiotic prophylaxis for IE have been formulated and updated frequently since they first appeared 
in 1955. IE is eight times high in patients with mitral valve prolapse than normal individuals. ${ }^{7}$ The 1997 AHA guidelines on IE recommended antibiotic prophylaxis to all high and moderate risk cardiac patients. But as time passed, the validity of the evidence behind the recommendation came under increasing scrutiny. In the 2007 guidelines, the committee toned down the recommendation for antibiotic prophylaxis to a small subset of patients. Patients with prosthetic cardiac valves or prosthetic material used for valve repair, previous infective endocarditis, uncorrected cyanotic congenital heart disease (CHD) including palliative shuts/conduits, corrected CHDs with prosthetic material or devices, residual defects adjacent to the site of prosthetic patch, cardiac transplant recipient developing cardiac valvulopathy/regurgitation are the high-risk groups when they undergo dental procedures commonly associated with bleeding/bacteremia. ${ }^{8}$ The committee recognized that unnecessary prophylaxis is potentially harmful for reasons like adverse drug reactions, antibiotic resistance and cost.

Intravenous antibiotics like aminoglycosides may affect renal function. All the four patients described in this series had received multiple antimicrobial agents and subsequently their blood urea levels increased up to 50 to $100 \mathrm{mg} \%$. Anemia and leukocytosis are the common features in infective endocarditis. Two patients had pallor with $\mathrm{Hb}$ of 7 and $9 \mathrm{gm} \%$ respectively. The unique association of our patients ( 2 out of 4 ) with type 2 DM has not been highlighted anywhere so far. Patient described in case 3 , although young had diabetes for over 3 years.

Large prospective randomized studies are lacking on IE, overall strategies are derived from retrospective series and clinical judgments. A multidisciplinary approach is recommended to optimize medical treatment with bactericidal antibiotics and decision making regarding indication and optimal timing of valve surgery. Often combined medical treatment and surgical valve replacement show a better outcome to avoid complications. ${ }^{9}$

The perioperative mortality in patients with IE depends upon the degree of left ventricular (LV) dysfunctions and damage to the cardiac tissues and the virulence of the infective agent at the time of surgery. ${ }^{10}$ Two patients had LV dysfunction with $\mathrm{EF}$ of 30 to $35 \%$. Induction with etomidate, liberal use of fentanyl and dobutamine infusion at weaning from $\mathrm{CPB}$ maintained hemodynamic stability. TEE played an important role in decision making during weaning phase. Patient, in case 3 , with severe MR, with hypermobile vegetation on AML was more challenging as she had systemic embolization of the left common iliac artery, external iliac artery and the femoral artery a couple of weeks prior to the surgery. Heparin infusion was started at the rate of $1000 \mathrm{U} /$ hour. Subsequently, she developed pleuropericardial temponade which was timely detected and efficiently managed. Patient (in case 1) with aortic root abscess could have developed atrioventricular (AV) block (as there is potential for AV nodal destruction in these subset of patients), therefore temporary pacing facilities were kept ready.

Intraoperative TEE is a useful tool for defining the IE lesions and its complications, like abscess and fistulae. TEE examination is recommended in patients with high clinical suspicion of IE and a normal transthoracic echocardiography (TTE) (class Ib). ${ }^{11}$ TEE has been found to be 82 to $96 \%$ sensitive versus 17 to $36 \%$ with TTE for diagnosis of IE. ${ }^{12}$ For follow-up and effect of antimicrobial therapy, repeat TEE is recommended if a new complication of IE is suspected (new murmur, embolism, persisting fever, heart failure, abscess, atrioventricular block) (class Ib). ${ }^{8}$ Overall, a comprehensive intraoperative TEE examination confirms the preoperative echo findings and may also provide additional information if focused examination pertaining to the diseased valve(s) is/are conducted. It also guides the surgeon to offer the best treatment option for the patient with valve lesion (like replacement versus repair). At the conclusion of surgery, it confirms the prosthetic valve function, assesses for any paravalvular leakage and looks for adequacy of valve repair, rules out excessive narrowing of the valve after repair and confirms obliteration of abscess cavity. In emergent situation like acute MR, acute AR with or without aoric dissection, acute Gerbode's defect or for that matter detecting any intra-cardiac lesion for the first time is an additional responsibility for the attending anesthesiologist. While executing these additional responsibilities, the anesthesiologist has to be diligent in preventing iatrogenic infections through meticulous aseptic precautions while handling the TEE probes. He should were sterile globes while introducing clean and sterile TEE probe. Antibiotic prophylaxis policy of the individual hospital should be formulated as per the latest guidelines and the prevalent flora of the medical centers.

\section{CONCLUSION}

The two most important things that cardiac anesthesiologists caring for patients with infective endocarditis can contribute are (1) meticulous aseptic technique and (2) a better and clearer picture of the heart for the surgeon to operate upon by intraoperative echocardiography with the help of 'TEE lenses'.

\section{REFERENCES}

1. Shapira Y, Weisenberg DE, Vaturi M, Sharoni E, Raanani E, Sahar G, Vidne BA, Battler A, Sagie A. The impact of intraoperative transesophageal echocardiography in infective endocarditis. Isr Med Assoc J 2007;9:299-302. 
2. Lopes S, Calvinho P, de Oliveira F, Antunes M. Allograft aortic root replacement in complex prosthetic endocarditis. Eur J Cardiothorac Surg 2007;32(1):126-130.

3. Miller B, Moore J, Mallon P, Xv J, Crowe M, McClurg R, Roult D, Earle J, Hone R, Murphy P. Molecular diagnosis of infective endocarditis - a new Duke's criterion. Scand J Inf Dis 2001;33: 673-680.

4. Blumberg EA, Karalis DA, Chandrasekharan K, Wahl JM, Vilaro J, Covalesky VA, Mintz GS. Endocarditis-associated paravalvular abscesses: Do clinical parameters predict the presence of abscess. Chest 1995;107:898-903.

5. Karalis DG, Bansal RC, Hauck AJ, Ross JJ Jr, Applegate PM, Jutzy KR, Mintz GS, Chandrasekaran K. Transesophageal echocardiographic recognition of subaortic complications in aortic valve endocarditis: clinical and surgical implications. Circulation 1992;86:353-362.

6. Daniel WG, Mugge A, Martin RP, Lindert O, Hausmann D, Nonnast-Daniel B, Laas J, Lichtlen PR. Improvement in the diagnosis of abscesses associated with endocarditis by transesophageal echocardiography. N Eng J Med 1991;324: 795-800.

7. Devereux RB. Recent developments in diagnosis and management of mitral valve prolapsed. Curr Prob Cardiol 1994; 16:369.

8. Wilson W, Taubert KA, Gewitz M, et al. Prevention of infective endocarditis: Guidelines from the american heart association: a guideline from the American Heart Association Rheumatic Fever, Endocarditis, and Kawasaki Disease Committee, council on cardiovascular disease in the young, and the council on clinical cardiology, council on cardiovascular surgery and anesthesia, and the quality of care and outcomes research interdisciplinary working group. Circulation 2007;116:1736-1754.

9. Vikram HR, Buenconrejo J, Hasbun R, et al. Impact of valve surgery at 6-month mortality in adults with complicated left sided native valve endocarditis: a propensity analysis. JAMA 2003;290:3207-3214.

10. David TE, Gavra G, Feindei CM, Regesta T, Armstrong S, Maganti MD. Surgical treatment of active infective endocarditis: a continued challenge. J Thoracic Cardiovasc Surg 2007; 133: 144-149.
11. Habib G, Hoen B, Tornos P, et al. Guidelines on the prevention, diagnosis and treatment of infective endocarditis (new version 2009). The task force on the prevention, diagnosis and treatment of infective endocarditis of the ESC. Eur Heart J 2009;30:23692413.

12. Murray MJ, Chapital AB, Giacobbe DT. Long-term complications and management. In: Kaplan JA, editor. Kaplan's Cardiac Anesthesia. Philadelphia: Saunders Elsevier 2011: p. 1091.

\section{ABOUT THE AUTHORS}

\section{Usha Kiran}

Professor, Department of Cardiac Anesthesia, All India Institute of Medical Sciences, New Delhi, India

\section{Arindam Choudhury (Corresponding Author)}

Assistant Professor, Department of Cardiac Anesthesia, All India Institute of Medical Sciences, New Delhi-110029, India, e-mail: archymd@gmail.com

\section{Neeti Makhija}

Professor, Department of Cardiac Anesthesia, All India Institute of Medical Sciences, New Delhi, India

\section{Kulbhushan Saini}

Senior Resident, Department of Cardiac Anesthesia, All India Institute of Medical Sciences, New Delhi, India

\section{Randhir Singh Rajput}

Senior Resident, Department of Cardiac Anesthesia, All India Institute of Medical Sciences, New Delhi, India

\section{Balram Airan}

Professor, Department of Cardiothoracic and Vascular Surgery, All India Institute of Medical Sciences, New Delhi, India 